\title{
Epiretinal membrane formation associated with spontaneous massive periretinal proliferation
}

\author{
ROGER MCGUINNESS \\ From the Department of Ophthalmology, University of New South Wales, \\ Kensington, New South Wales, Australia
}

SUMMARY A 74-year-old man with cardiovascular disease and epiretinal membrane formation developed spontaneous massive periretinal proliferation over a period of 3 months. No surgery was performed at any stage. A 3-year follow-up is described and theories concerning the production of + these 2 entities are discussed.

Epiretinal membrane formation is not uncommon in an older population, appearing first as a glistening reflex from the surface of the retina, more easily seen in red-free light (Wise, 1975). As the membrane thickens and retracts, superficial retinal folds appear and macular pucker can occur. Massive periretinal proliferation, formerly called massive vitreous or preretinal retraction, may follow operations for retinal detachment.

The following case history describes a continuing process of epiretinal membrane formation leading to spontaneous massive periretinal proliferation without any surgical procedure being performed.

\section{Case report}

A 74-year-old man suffering from cardiovascular disease presented with deterioration of vision in his left eye. On examination visual acuities were 6/9 right and 6/36 left, with a spectacle correction. The right eye was normal, but the left showed several flame-shaped haemorrhages near the optic disc margin, with an epiretinal membrane over the central area, giving the appearance of oedema (Fig. 1). No vitreous cells, pigment, or detachment were present. Fluorescein angiography showed early leakage of fluorescein from the disc and temporal vessels and some staining of the vessel walls (Fig. 2).

The patient was seen at intervals, and some 3 months after the initial consultation he presented with a history of loss of vision in his left eye of 2 days' duration. On examination visual acuities were $6 / 6$ right, hand movements left. The vitreous showed

Correspondence to Dr Roger McGuinness, Department of Ophthalmology, University of New South Wales, Prince of Wales Hospital, Randwick, New South Wales 2031, Australia.

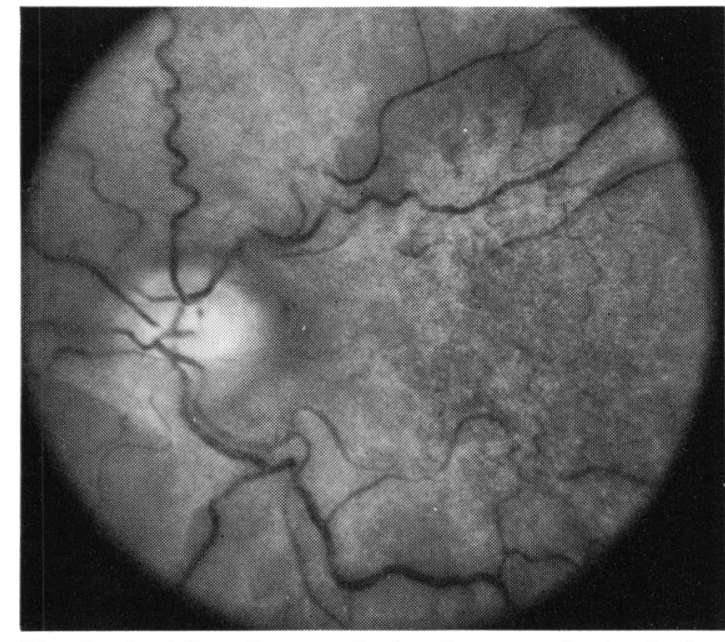

Fig. 1 Red-free photograph showing central epiretinal membrane

a large number of pigment clumps just anterior to an extensive retinal detachment. The retina showed $\stackrel{\circ}{5}$ greyish folds with 2 tears in the upper temporal $>$ quadrant (Fig. 3). The patient declined operation, and over the next few days the retina rapidly con- N tracted into a funnel-shaped retinal detachment showing the typical appearance of periretinal $\sim$ proliferation (Fig. 4).

Over a period of 1 year there was considerable ${ }^{O}$ large pigment clumping behind the lens in the region ${ }^{\circ}$ of the ligament of Wieger and just in front of the retina. A complete membrane existed across the retina, including the central funnel leading to the $\frac{0}{0}$ optic disc, which could not be clearly seen. The $\frac{}{\mathrm{D}}$ original retinal tears had contracted and were $\frac{\rho}{\Phi}$ covered by a membrane containing new vessels. 


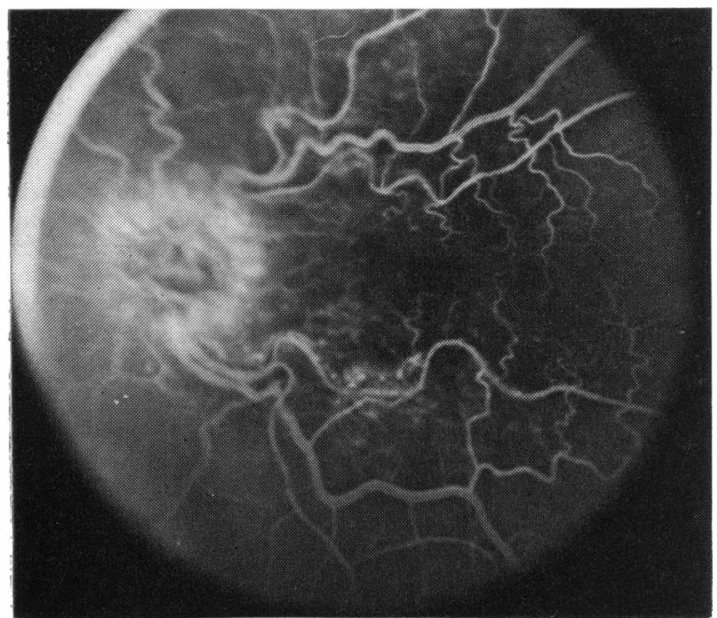

Fig. 2 Fluorescein angiogram showing early leakage of fluorescein from the disc and temporal retinal vessels and some staining of the retinal vessel walls

Two years after the onset of the massive periretinal proliferation the pigment began to disappear. After 3 years vision was no perception of light in the left eye, and the pigment at the back of the lens and on the retina had practically disappeared. The membrane over the funnel leading to the optic disc had retracted, and the optic disc could now be seen. The original retinal tears in the upper temporal quadrant were obscured by fibrous tissue across the surface, but the new vessels were still present.

\section{Discussion}

Epiretinal membrane formation has been thought to follow cell proliferation. This proliferation may be from retinal pigment epithelial cells (Machemer and Laqua, 1975) or Muller cells and haematogenous macrophages (Gloor and Daiker, 1975) or astrocytes (Foos and Gloor, 1975). Inflammation may also be a factor, and in some cases epiretinal membrane formation may follow vasculitis. Breaks in the inner limiting membrane of the retina have been implicated (Foos, 1972).

The present case shows that epiretinal membrane formation and periretinal proliferation can form a continuous process. The opinion that spontaneous retinal pucker never leads to massive periretinal proliferation is not correct on the evidence of the present case (Machemer, 1978). It is of interest that the patient did not develop pigment in the vitreous until the stage at which the retina was detached and in fixed folds.

It would appear that if there is enough fibro-

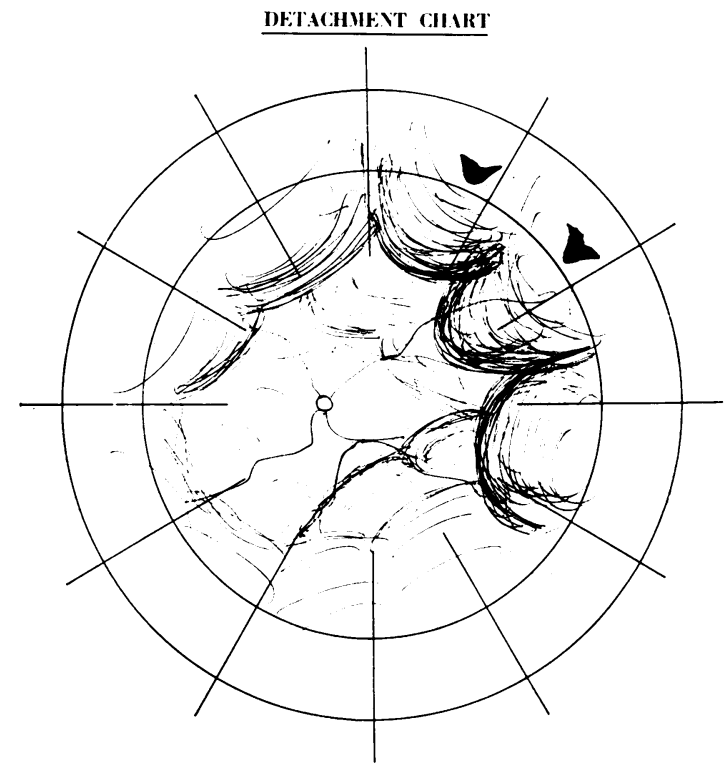

Fig. 3 Fundus drawing showing retinal detachment with folds and 2 tears in the upper temporal quadrant

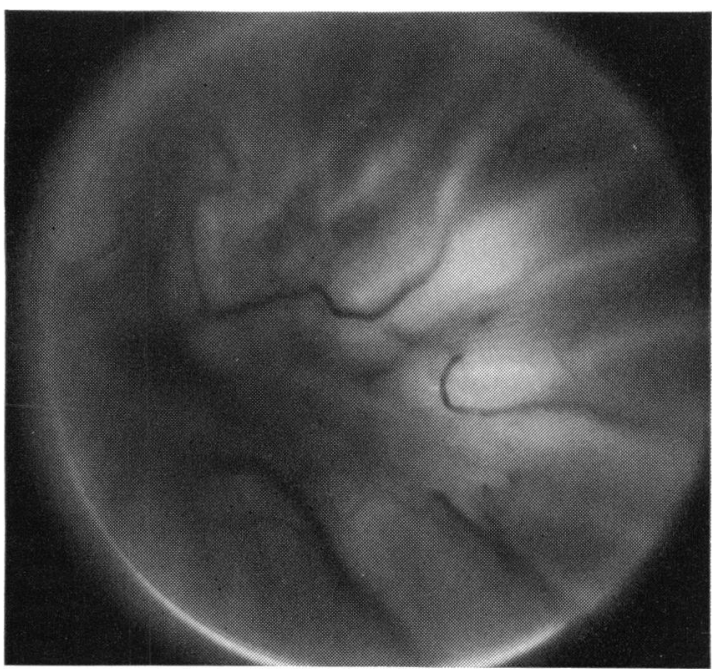

Fig. 4 Fundus photograph showing a funnel-shaped retinal detachment typical of periretinal proliferation

cellular proliferation on the surface, then the retina is capable of being rucked up and retinal tears can be produced. The role of vitreous contraction in this process is uncertain, and in the present case a posterior vitreous detachment was not present at any stage, so that any vitreous contraction could directly affect the retina.

I am grateful to Dr C. N. Banks for his original referral of the case, Dr Paul Mitchell for performing the fluorescein 
angiogram, and Mr Ken Deason and the staff of the Medical Illustration Department of the Prince of Wales Hospital, Randwick, for the illustrations.

\section{References}

Foos, R. Y. (1972). Vitreoretinal juncture: topographical variations. Investigative Ophthalmology, 11, 801-808.

Foos, R. Y., and Gloor, B. P. (1975). Vitreoretinal juncture: healing of experimental wounds. Albrecht von Graefes Archiv für klinsche und experimentelle Ophthalmologie, 196, 213-220.

Gloor, B. P., and Daiker, B. C. (1975). Pathology of the vitreo-retinal border structures. Transactions of the Ophthalmological Societies of the United Kingdom, 95, 387-390.

Machemer, R. (1978). Pathogenesis and classification of massive periretinal proliferation. British Journal of Ophthalmology, 62, 737-747.

Machemer, R., and Laqua, H. (1975). Pigment epithelial proliferation in retinal detachment (massive periretinal proliferation). American Journal of Ophthalmology, 80, 1-23.

Wise, G. N. (1975). Clinical features of idiopathic preretinal macular fibrosis. American Journal of Ophthalmology, 79, 349-357. 